\title{
Research of a corrosion condition of an inventory of oil refineries on the example of complex works on monitoring of corrosion rate of process pipelines
}

\author{
Oleg Kiselev ${ }^{1, *}$ \\ ${ }^{1}$ AO VNIKTINHO, Laboratory of Analytical Chemistry and Corrosion, 400087 Volgograd, Russia
}

\begin{abstract}
In article the modern methods of corrosion monitoring which is one of the main tools for assessment of corrosion rate of processing equipment during its production activity are considered. Results of researches of corrosion processes and the analysis of corrosion rates of processing equipment of oil refineries are given, and the conclusion is also drawn on effectiveness of corrosion monitoring by a gravimetric method for monitoring of process of corrosion of an inventory in the conditions of aggressive environments.
\end{abstract}

Now studying of corrosion processes of the inventory used at oil refineries and its corrosion protection are one of the major problems. Assessment of corrosion rates of processing equipment is applied to an effective solution of similar tasks during its production activity (corrosion monitoring). Today the main methods of corrosion monitoring are gravimetric, the linear polarizing resistance (LPR) and electrical resistance (ER) [1].

The method of the linear polarization serves for monitoring as deviations of aggression of the environment which can happen because of jump of serviceable conditions or violations in technology of inhibition. The scheme of placement of the LPR sensor in the pipeline is provided on figure 1.

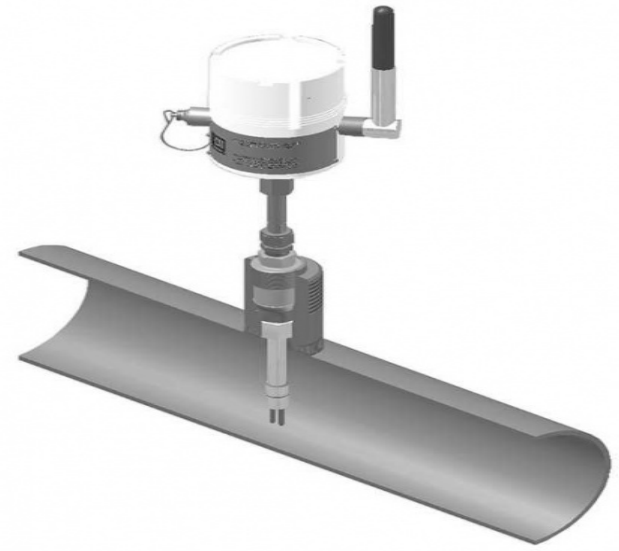

Fig. 1. The scheme of placement of the LPR sensor in the pipeline.

Advantage of this method is the high sensitivity to changes of the modes of production activity: to temperature, pressure, the maintenance of aggressive components etc. that allows to react in real time to change of corrosion processes of an inventory. However, the LPR method has a number of restrictions: it is used only in well conducting environments to which the aqueous phase, a water oil emulsion with water content not less than $40-50 \%$ belong; distortion of results owing to formation of sulphidic deposits on the sensor.

The method of electrical resistance also serves for monitoring as changes of aggression of a working fluid as a result of deviations of parameters of the mode of production activity and technology of inhibitory protection in real time. As against the LPR method the ER method is applicable both for the fluids which are well carrying electric current and with low conduction. The main problem of this method is decrease in accuracy of the received results of corrosion rate owing to accumulation on a surface of a sensing device of the sensor of corrosion deposits.

The gravimetric method allows to define loss of mass of metal specimens during their stay in aggressive examinees fluids with the subsequent assessment of corrosion rate [2]. Besides this method fully represents character and features of corrosion damages of metal. Specimens from material of an inventory similar to material are usually applied to assessment. For realization of a gravimetric method under operating conditions of the inventory working under pressure devices for input of specimens (coupons) in an actuation medium and, after endurance of specimens during the allowed time, for their extraction from an actuation medium are used. Such devices are called corrosion probes (coupon holders). The appearance of such device is presented in figure 2. The specimens used for realization of a gravimetric method are subject to special processing both before installation in an actuation medium, and after extraction from an actuation medium.

* Corresponding author: kiselevos@,vniktinho.ru 


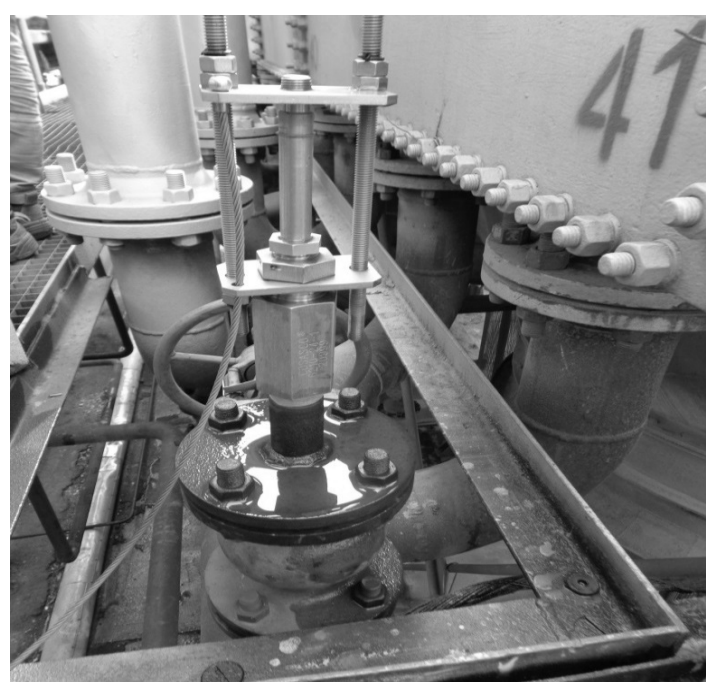

Fig. 2 Appearance of the coupon holder.

Because application of the ER and LPR methods a number of restrictions have, the bound to accumulation on a surface of a sensing device of the corrosion deposits distorting data, the purpose of work is the research of corrosion processes and the analysis of corrosion rates of processing equipment of the oil refineries based on application of a gravimetric method of corrosion monitoring.

Comprehensive measures on monitoring of corrosion rate of an inventory were taken by means of placement of the specimens witnesses in process pipelines made of carbon steel (19 pieces), austenitic chromium-nickel stainless steel (5 pieces) and brass (1 piece), on 12 oil refinery units (primary petroleum refining, hydrotreating, catalytic reforming, cracking, etc.). Besides collecting the data about controlled indexes of actuation mediums affecting corrosion aggression and the applied methods of influence on it (neutralization, inhibition) was carried out. It allows to estimate not only corrosion rate, but also to make check on tendency of materials of an inventory to local corrosion, corrosion cracking, intercrystalline corrosion, and if necessary, check of rust resistance of other materials. Realization of corrosion monitoring at oil refinery was carried out for the purpose of monitoring procedure of behavior of corrosion processes and quality of anticorrosive protection with the subsequent readout of recommendations about elimination of the causes of corrosion.

The analysis of the obtained data during tests from September, 2016 to March, 2019 showed that on the majority of controlled pipelines of process units general uniform corrosion with a speed which isn't exceeding normative $-0.1 \mathrm{~mm} /$ year is observed. However several pipelines at which during various periods of tests deviations from standard values of corrosion rate are recorded were noted. So on lines of units of primary petroleum refining of atmospheric distillation unit (ADU), atmospheric vacuum distillation unit (ADU/VDU) and the hydrotreating of vacuum gasoil (HVG) short-term increase in speed to $0.36,0.17$ and 0.8 $\mathrm{mm} /$ year respectively is revealed. Besides the appearance of specimens witnesses showed existence of signs of origin of pit corrosion (figure 3 ).

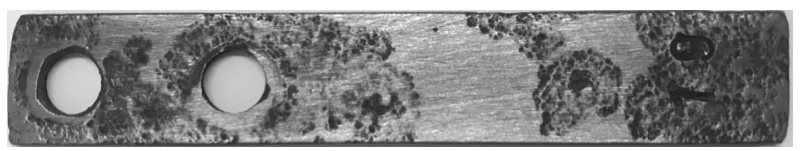

Fig. 3 Specimen witness of the vapor line of a column of stabilization of ADU/VDU after removal of corrosion deposits.

Studying of the reasons of the raised corrosive wear for vapor pipelines of columns of stabilization of units of primary petroleum refining from carbon steel, under the influence of the fluid consisting of gasoline and water showed that increase in corrosion rates is bound to the low-temperature hydrogen-sulfide corrosion what a large amount of sulfides in actuation mediums testifies to (up to $300 \mathrm{mg} / \mathrm{dm}^{3}$ ). Besides jumps of content of chlorides over $40 \mathrm{mg} / \mathrm{dm}^{3}$ are recorded that can lead to failure of inhibitory protection. For these pipelines, and also for the pipeline of the stripped water of sour drains of HVG it was succeeded to reduce corrosion rates to normative values after control of the maintenance of aggressive components in actuation mediums and effectiveness of inhibitory protection.

Tests of specimens witnesses on the line of the regenerated solution of methyldiethanol amine (MDEA) of HVG showed that the high speed of corrosion during some periods developing $1.5 \mathrm{~mm} /$ year was for an appreciable length of time observed. On a surface of these specimens made of carbon steel after extraction from an actuation medium, existence of pronounced pit corrosion is recorded (figure 4).

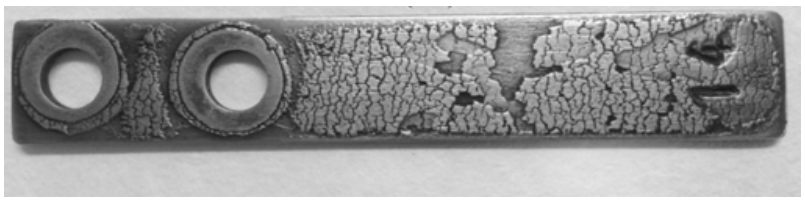

Fig. 4. Specimen witness of the MDEA pipeline of the HVG unit after removal of corrosion deposits.

Comparison of the data on corrosion rate received on every period of tests of specimens from the considered pipeline showed that the aggression of the regenerated MDEA solution continuously increased eventually. Increase of corrosion aggression of solutions of ethanolamines is bound to their gradual degradation, the bound to oxidation and formation of corrosive agents. During the cleaning of gases there occur reactions which result in formation of side compounds (formamides, amino acids, oxazolidones, urea, diamines). The gases subjected to cleaning by means of ethanolamines may contain a great quantity of various impurities which irreversibly react with amines: sulphur compounds, carbonic acids, etc. With all these compounds amines form simple thermally stable salts, for example, such as thiosulfates, resulting from interaction of ethanolamine solutions with $\mathrm{H} 2 \mathrm{~S}$; N-oxyethylthiocarbamino acid 
which is a product of the reaction between monoethanolamine and carbon sulphooxide; oxazolidones, diamines (N-(2-oxyethyl)-ethylenediamine) which are produced as a result of irreversible reaction of ethanolamines with $\mathrm{CO} 2$. Different factors affect the formation of oxazolidones: concentration of carbon dioxide in the initial gas, solution saturation, amount of the solution which circulates through the unit, operating conditions, etc. [3].

External signs of increase of corrosion activity of MDEA solution are: change of its color from colourless to black, increase in viscosity and decrease $\mathrm{pH}$. After implementation of the recommendations for the presented process pipeline including cleaning of oxidates and degradations of MDEA solution, check of effectiveness of an inhibitor, corrosion rate no more than $0.1 \mathrm{~mm} /$ year. gradually decreased to normative values that is visible from figure 5 .

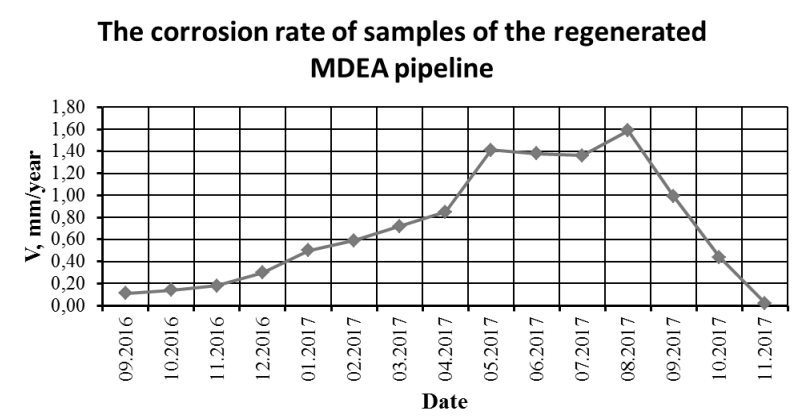

Fig. 5. Change of corrosion rate of specimens witnesses of the line of the regenerated MDEA during various periods of tests.

Monitoring of corrosion rate by means of realization of a gravimetric method and recommendations provided on the basis of its results allows to reduce the volume of repair work on process units that is the most important factor of an economic component of all process. Results of the current corrosion monitoring show a high significance and effectiveness of a gravimetric method for monitoring of process of corrosion steels in aggressive environments in the conditions complicated by processes of formation of corrosion deposits.

The main problem of this method is the impossibility of data acquisition about change of aggression of the environment, corrosion rate, corrosion processes in real time. Therefore, a comprehensive approach with application of all above-mentioned control methods of corrosion processes is necessary for the most effective corrosion monitoring that in total with application of a complex of methods of nondestructive monitoring will allow to exercise in real time control of corrosion of an inventory and pipelines on the scale of oil refinery. Collaboration of the experts who are qualified in the field of the corrosion necessary for interpretation of data on current state of materials of an inventory is necessary for ensuring effective work and realization of all opportunities of a similar system both from research and design institutes, and from productions.

\section{References}

1. A.N. Monakhov, A.K. Kuznetsov, M.A. Monakhova, Experience of using corrosion sensors in corrosion monitoring systems (Exposition Oil\&Gas, 2015, №2)

2. P.A. Lobova, A.N. Baranov, Corrosion monitoring as a means to control the pipeline integrity in petrochemical industry (Systems. Methods. Technologies, 2014, №3)

3. S.N. Shirokov, V.I. Geraskin, V.A. Emelkina, M.S. Alimova, E.A. Mirovskaya, Combating corrosion in amine systems (Exposition Oil\&Gas, 2008, №5) 